\title{
Light Emission from CdS Quantum Dots Stabilized by Sugars
}

\author{
I. Sergiel*, A. Mirończyk, J.J. Koziol And A. Defort \\ Department of Biotechnology, Faculty of Biological Sciences, University of Zielona Góra \\ Prof. Z. Szafrana 1, 65-516 Zielona Góra, Poland
}

\begin{abstract}
CdS nanoparticles were successfully synthesized using starch, amylopectin and maltodextrin, as a capping agent. Optical properties of the final products have been investigated by photoluminescence spectroscopy and Raman scattering. The atomic force microscopy gave a particle of an average size of approximately $6 \mathrm{~nm}$. Our research reveals that all prepared sugar-quantum dot aqueous solutions were stable against precipitation during the time of their investigation and kept their stability for several months after it.
\end{abstract}

PACS numbers: 73.21.La, 78.67.Hc, 78.55.Et

\section{Introduction}

Semiconductor quantum dots (QDs) have been the subject of great scientific and technological interest because of their remarkable optical and electronic properties, as well as potential for a wide range of applications [1-3]. Optical properties render QDs ideal fluorophores for ultrasensitive and multiplexing applications in cell and molecular biology as well in bioengineering $[4,5]$. In 1998 two groups, one led by Alivisatos at UC-Berkeley and another led by Nie (Indiana University, Bloomington), simultaneously demonstrated that QD could be made soluble in water and could be conjugated with biological molecules [6, 7].

One of the most popular choices of QDs materials is CdS. Cadmium sulfide is most often synthesized through the combination of cadmium and sulfur precursors in the presence of a QD-binding ligand that stabilizes the growing QD particles and prevents their aggregation into bulk semiconductors. In recent papers many suitable capping agents, with such saccharides, among them lactose, melibiose, maltotriose, chitosan and starch, have been studied to obtain CdS (QDs) [8-12]. A critical problem in the application of QD nanoparticles in biological labeling is related to their size stability upon aging [13]. For biological application, the QDs must be coated with a biocompatible polymer to improve photostability properties and minimize the toxic side effects. Moreover a large amount of literature that include the studies of optical properties of nanosized cadmium sulphide have been published by several groups, however there are only few reports on high stability of CdS (QDs) in air (Khanna et al. described synthesis and optical studies of $\mathrm{CdS}$ in N, $\mathrm{N}^{\prime}$ -dimetylformamide and in polymethylmethacrylate) [14].

\footnotetext{
* corresponding author; e-mail: I.Sergiel@wnb.uz.zgora.pl
}

The purpose of the present work is preparation of CdS nanoparticles using starch, amylopectin and maltodextrin as capping agents in aqueous solution and studying of their stability in air. In our research, these polysaccharides were selected as a protecting agent because they are renewable and they are a good dispersion medium to form stable CdS. Besides, many researchers have shown that hydroxyl groups of polysaccharides act as a passivation center for stabilization of the nanoparticles [11]. Our results clearly indicated, that the solutions of CdS (QDs) were reasonably stable in air. These particles should be good enough for useful applications. The obtained novel sugar-QDs (starch-QD, amylopectin-QD and maltodextrin-QD) is indeed a remarkable achievement of the present work.

\section{Experimental procedures}

Cadmium sulfide nanoparticles were prepared by mixing cadmium nitrate $\left(\mathrm{Cd}\left(\mathrm{NO}_{3}\right)_{2}\right)$, potassium hydroxide $(\mathrm{KOH})$, ammonium nitrate $\left(\mathrm{NH}_{4} \mathrm{NO}_{3}\right)$ and thiourea $\left(\mathrm{CS}\left(\mathrm{NH}_{2}\right)_{2}\right)$. Soluble starch, amylopectin and maltodextrin were used as a capping agent. All reagents were of commercially available materials and were used as received. Deionized water (DI water) was used as the solvent. Polysaccharides capped nanoparticles were prepared through a modified process described in the literature [15].

In this procedure $110 \mathrm{ml}$ of DI water was mixed with $0.073 \mathrm{~g}$ of cadmium nitrate, $2.4 \mathrm{~g}$ of ammonia nitrate, $1.4 \mathrm{~g}$ of potassium hydroxide and $0.304 \mathrm{~g}$ of thiourea. The thiourea was added after $10 \mathrm{~min}$ since the beginning of the process. The mixture was stirred and heated at $80^{\circ} \mathrm{C}$. Similar conditions were applied to starch, maltodextrin and amylopectin in $1 \mathrm{~g} / 100 \mathrm{ml}$ concentration. These solutions were slowly added into a flask. The mixture was kept at the temperature of $80^{\circ} \mathrm{C}$ and maintained at this 
temperature for $30 \mathrm{~min}$. Solutions turned yellow indicating the initial formation of $\mathrm{CdS}$ nanoparticles. The global reaction towards CdS formation can be summarized as

$$
\begin{aligned}
& \mathrm{Cd}\left(\mathrm{NH}_{3}\right)_{4}\left(\mathrm{NO}_{3}\right)_{2}+\mathrm{SC}\left(\mathrm{NH}_{2}\right)_{2}+2 \mathrm{KOH} \\
& \quad \rightarrow \mathrm{CdS}+\mathrm{CH}_{2} \mathrm{~N}_{2}+4 \mathrm{NH}_{3}+2 \mathrm{KNO}_{3}+2 \mathrm{H}_{2} \mathrm{O} .
\end{aligned}
$$

The photoluminescence spectra of clear solutions of CdS (QDs) were achieved using Perkin Elmer LS 55 fluorescence spectrophotometer with $350 \mathrm{~nm}$ excitation line.

Raman spectra of the powder nanoparticles were recorded using a Raman FRA 106 spectrometer employing the $1060 \mathrm{~nm}$ line from Nd:YAG laser.

Atomic force microscopy (AFM) observation was conducted using AFM (NTEGRA, NT-MDT). The sample was dipping coated on the fresh-mica surface directly from the solution, and then measured by AFM in semicontact mode. The cantilever, that has been used here, has a constant force of $5.5 \mathrm{~N} / \mathrm{m}$, and frequency about $250 \mathrm{kHz}$, with a $10 \mathrm{~nm}$ diameter tip.

\section{Results}

We examined the emission spectra of starch-, maltodextrin- and amylopectin-CdS nanoparticles in aqueous solutions following 100 days of storage. The results are shown in Fig. $1 \mathrm{~A}-\mathrm{C}$, respectively. As may be observed from these figures, aqueous solutions of CdS QDs were stable over a long period of time and showed no changes in optical properties. The aged samples indicated that the size of the sugar-QDs remained practically unaffected following storage.

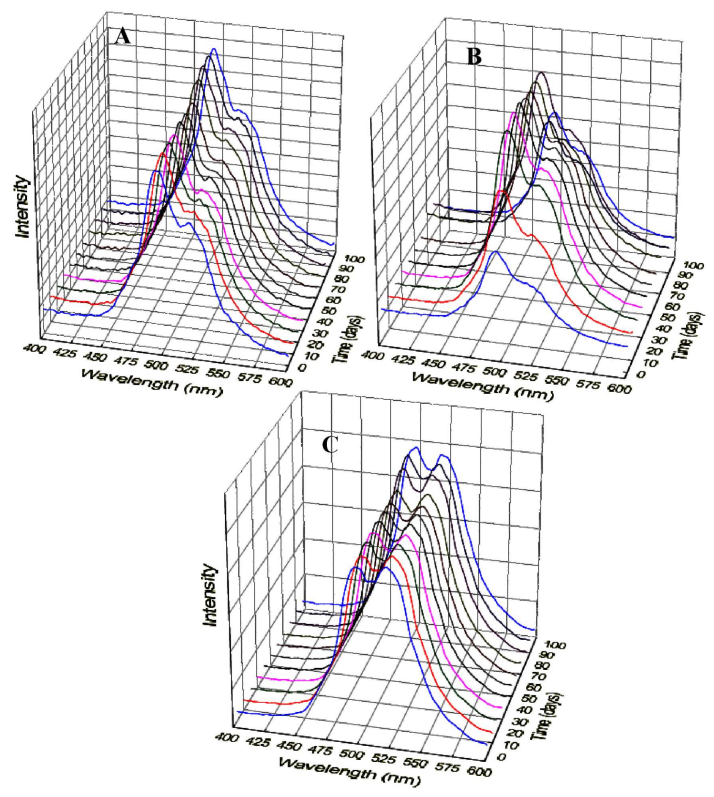

Fig. 1. Photoluminescence emission spectra of sugar capped CdS nanoparticles: (A) starch-QD, (B) maltodextrin-QD, (C) amylopectin-QD, following 100 days of storage.
The figures show that at excitation wavelength at $350 \mathrm{~nm}$, two emission bands (one peak at $495 \mathrm{~nm}$ indicates a particle size of about $6 \mathrm{~nm}[16,17]$ and another at $520 \mathrm{~nm}$ ) may be observed. $\mathrm{Fu}$ et al. have also reported two luminescence bands for CdS, i.e. blue and green [18]. The high intensity band at $495 \mathrm{~nm}$ is due to exciton recombination in the mostly delocalized states in nanocrystals (the band-edge emission) and low intensity peaks at $520 \mathrm{~nm}$ may result from recombination via surface-localized states (the trap-state emission) (Figs. 1 and 2) [19]. Fluorescence spectrum of amylopectin capped CdS shows two bands similarly as the spectra with starch and maltodextrin, but the position of the long wavelength band (Fig. 2) is red-shifted at deposition time (100 days). The reason for his effect might be a lower lying equilibrium excited state or higher FranckCondon ground state in the case of amylopectin capped CdS.



Fig. 2. Photoluminescence emission spectra of uncapped CdS (blue), starch-CdS (green), maltodextrinCdS (red) and amylopectin-CdS (purple).

In contrast, uncapped CdS in our samples also exhibit two bands around $490 \mathrm{~nm}$ and $530 \mathrm{~nm}$ (Fig. 2), however, our spectral results show very weak deep trap-state emission and band-edge emission for bare CdS.

Kumar et al. showed that surface passivation removes the trap state, therefore, the density of trap state of capped CdS QDs should be lower than those of the uncapped samples; this is in agreement with our experimental results [20].

In our case the hydroxyl groups act as passivation contacts for the stabilization of the CdS nanoparticles in the aqueous solution. The observed high stability is mainly due to the polyelectrolyte property of sugars.

Raman spectra of the starch and maltodextrin capped CdS nanoparticles are shown in Fig. 3A,B.

The spectrum of the starch capped CdS exhibits a peak at $300 \mathrm{~cm}^{-1}$ and its second harmonics around $600 \mathrm{~cm}^{-1}$. These results remain in agreement with the Raman spectra from the literature $[11,21]$.

For maltodextrin CdS nanoparticles, we found also two peaks characteristic for $\mathrm{CdS}$-polysaccharides that are at $300 \mathrm{~cm}^{-1}$ and at $600 \mathrm{~cm}^{-1}$. 




Fig. 3. Raman spectra of (A) starch-CdS (QD) (blue) and starch (red), (B) maltodextrin-CdS (QD) (blue) and maltodextrin (red), show the LO optical phonon (at $300 \mathrm{~cm}^{-1}$ ) and its second harmonic (at $600 \mathrm{~cm}^{-1}$ ) for sugar-CdS (QDs).

The Raman bands at 576, 478, and $440 \mathrm{~cm}^{-1}$ are attributed to the skeletal mode vibrations of the glucose pyranose ring [11]. Associating all those results, we can seen that the degree of polymerization in starch capped CdS-QDs is higher than in maltodextrin-CdS-QDs.

Figure 4A,B presents AFM images of starch-CdS and amylopectin-CdS nanoparticles. The estimated size of the nanoparticle of about $6 \mathrm{~nm}$ is similar to that obtained in PL experiments.



Fig. 4. AFM images of starch-CdS (A) and amylopectin-CdS (B) nanoparticles.

\section{Summary}

In summary, we have successfully synthesized the novel cadmium sulfide nanoparticles using starch, maltodextrin, and amylopectin as capping agents. Moreover, the paper describes studies of optical properties of CdS nanoparticles. Our aqueous solutions of CdS QDs were stable over a long period of time and showed no significant change in optical properties even after a few months of exposure to the day light. The size of the particle was obtained from photoluminescence and AFM characteristics.

\section{References}

[1] K.K. Nanda, S.N. Sarangi, S. Mohanty, S.N. Sahu, Thin Solid Films 322, 21 (1998).

[2] O. Vigil, Y. Rodrìquez, O. Zelaya-Angel, C. Vazquez-Lòpez, A. Morales-Acevedo, J.G. Vàzquez-Luna, Thin Solid Films 322, 329 (1998).

[3] M. Green, P. Brien, Chem. Commun. 1999, 2235 (1999).

[4] X. Michalet, F.F. Pinaud, L.A. Bentolila, J.M. Tsay, S. Doose, J.J. Li, G. Sundaresan, A.M. Wu, S.S. Gambhir, S. Weiss, Science 307, 538 (2005).

[5] A.M. Smith, X. Gao, S. Nie, Photochem. Photobiol. 80, 377 (2004).

[6] W.C.W. Chan, S. Nie, Science 281, 2016 (1998).

[7] M. Bruchez Jr., M. Moronne, P. Gin, S. Weiss, A.P. Alivisatos, Science 281, 2013 (1998).

[8] P. Babu, S. Sinha, A. Surolia, Bioconjugate Chem. 18, 146 (2007).

[9] B. Kang, S. Chang, Y. Dai, D. Chen, Radiat. Phys. Chem. 77, 859 (2008).

[10] S. Sadhu, P.S. Chowdhury, A. Patra, J. Lumin. 128, 1235 (2008).

[11] P. Rodriquez, N. Muñoz-Aguirre, E.S. Martinez, G. González de la Cruz, S.A. Tomas, O. Zelaya-Angel, J. Cryst. Growth 310, 160 (2008).

[12] O. Wei, S. Kang, J. Mu, Coll. Surf. A, Physicochem. Eng. Aspects 247, 125 (2004).

[13] D. Kaushik, R. Raj Singh, M Sharma, D.K. Gupta, N.P. Lalla, R.K. Pandey, Thin Solid Films 515, 7070 (2007).

[14] P.K. Khanna, R. Gokhale, V.V.V.S. Subbarao, Mater. Lett. 57, 2489 (2003).

[15] A.I. Oliva, R. Castro-Rodríquez, O. Ceh, P. Bartolo-Pérez, F. Caballero-Briones, V. Sosa, Appl. Surf. Sci. 148, 42 (1999).

[16] A. Henglein, Chem. Rev. 89, 1861 (1989).

[17] J.M. Costa-Fernández, R. Pereiro, A. Sanz-Medel, Trends Anal. Chem. 25, 207 (2006).

[18] Z. Fu, S. Zhou, J. Shi, S. Zhang, Mater. Res. Bull. 40, 1591 (2005).

[19] F. Wu, J.Z. Zhang, R. Kho, R.K. Mehra, Chem. Phys. Lett. 330, 237 (2000).

[20] A. Kumar, E. Janata, A. Henglein, J. Phys. Chem. 92, 2587 (1988).

[21] J. Lee, Thin Solid Films 451, 170 (2004). 